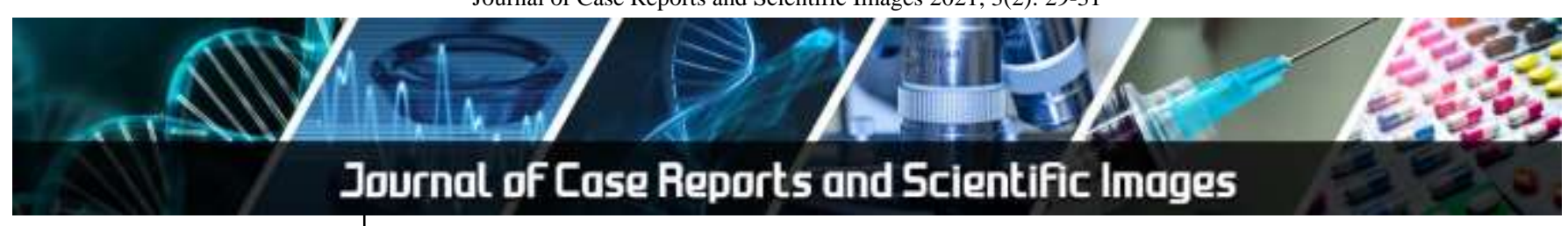

E-ISSN: 2708-0064

P-ISSN: 2708-0056

IJCRS 2021; 3(2): 29-31

http://www.allcasereports.com

Received: 16-06-2021

Accepted: 25-07-2021

Das Uddalok

Post Graduate Trainee (batch of 2020-2023), Department of Radiodiagnosis NBMCH, West Bengal, India

Selim Sahajada

Post Graduate Trainee (batch of 2019-2022), Department of Radiodiagnosis NBMCH, West Bengal, India

Singh Ramudar

Post Graduate Trainee (batch of 2019-2022), Department of Radiodiagnosis NBMCH, West Bengal, India

Pandit Narayan

Professor and Head of Department, Department of Radiodiagnosis NBMCH, West Bengal, India

\section{Eventration of diaphragm masquerading as a congenital diaphragmatic hernia in a neonate}

\author{
Das Uddalok, Selim Sahajada, Singh Ramudar and Pandit Narayan
}

DOI: https://doi.org/10.22271/27080056.2021.v3.i2a.36

\begin{abstract}
Eventration of the diaphragm is a condition wherein the diaphragm is abnormally elevated while retaining its normal attachments to the sternum, ribs, and dorsolumbar spine. The continuity of the diaphragm remains intact. This is a rare anomaly and is often mistaken as a diaphragmatic hernia on chest radiographs by physicians. Magnetic Resonance Imaging (MRI) gives a definitive diagnosis. We present the case of a neonate who was admitted to the Neonatal Intensive Care Unit and after X-ray and ultrasonography (USG) was presumed to have a right-sided congenital diaphragmatic hernia. But then MRI was done and the diagnosis was changed to eventration of the diaphragm as the continuity of the diaphragm was maintained. The baby was treated with surgery.
\end{abstract}

Keywords: Diaphragmatic hernia, neonate, sternum, ribs, and dorsolumbar spine

\section{Introduction}

Eventration of the diaphragm is a rare disorder. All or a part of the diaphragmatic muscle is replaced by fibro-elastic tissue. The diaphragm retains its continuity and attachments to the costal margin and sternum. Due to weakness of the diaphragm, it can displace the thorax which causes respiratory compromise. It is a rare anomaly with an incidence of 5 in 10,000 live births ${ }^{[1]}$. Eventration of the diaphragm can be congenital or acquired ${ }^{[2,3]}$. We report this rare condition in a term female neonate who presented with respiratory distress at 8 hrs of life and was first misdiagnosed as a right-sided congenital diaphragmatic hernia based on XRay. However, after Magnetic Resonance Imaging (MRI) was done the diagnosis was changed into eventration of the diaphragm.

\section{Case report}

A $2.1 \mathrm{~kg}$ term female neonate was born to 25 years old primigravida lady at term by normal vaginal route. The mother was from a poor background and did not have any ultrasonography examination during her antenatal period. The baby did not cry immediately after birth. APGAR score at 1 min was 4/10. The baby was resuscitated with bag and mask ventilation and at 5 min APGAR score was 7/10. The baby was kept at the High Dependency Unit for observation. The. At $8 \mathrm{hrs}$ of life, the baby developed poor feeding and fast breathing and was shifted to the Neonatal Intensive Care Unit. The vital parameters revealed a heart rate of 140 per minute, afebrile, respiratory Rate 68/minute with no retractions, noninsvasive blood pressure (NIBP) 66/40 mm Hg, SpO2 90\% room air, and 95\% with oxygen, capillary filling time $<2$ seconds, and all peripheral pulses were palpable. The systemic evaluation revealed a decrease in air entry on right. The heart sound was shifted to the left. The other system was essentially normal. The laboratory investigation were all within normal limits. Emergency ultrasonography (USG) and Chest X-ray were done. USG revealed the position of the liver high up in the thorax at a plane near the plane of the heart (Figure-1). Chest x-ray AP supine revealed grossly elevated right hemidiaphragm with decreased right lung volume and encroachment of liver and bowel loops into the thorax (Figure-2). A provisional diagnosis of right-sided congenital diaphragmatic hernia was made. After the initial stabilization of the patient, the case was referred to the Radiodiagnosis department for an expert opinion. On suspicion of the examining radiologist, an MRI scan of the abdomen and thorax was done after sedating the baby. MRI revealed gross elevation of the right hemidiaphragm along with the relatively higher position of the liver. No breach or discontinuity in the contour of the diaphragm could be demonstrated. There was no decrease in right lung volume. No tracheal or mediastinal shift could be noted (Figure-3). The diagnosis was changed into eventration of right hemidiaphragm.
Corresponding Author: Das Uddalok

Post Graduate Trainee (batch of 2020-2023), Department of Radiodiagnosis NBMCH, West Bengal, India 
The neonate was managed with IV fluids, antibiotics, CPAP with PEEP $5 \mathrm{~cm}$ and $\mathrm{FiO} 250 \%$ which was gradually weaned to oxygen hood, and the baby was planned for definitive surgery. Surgical plication of the right hemidiaphragm was done by pediatric surgeons. The postoperative period was uneventful. The baby is kept under regular follow-up by the Surgery department.

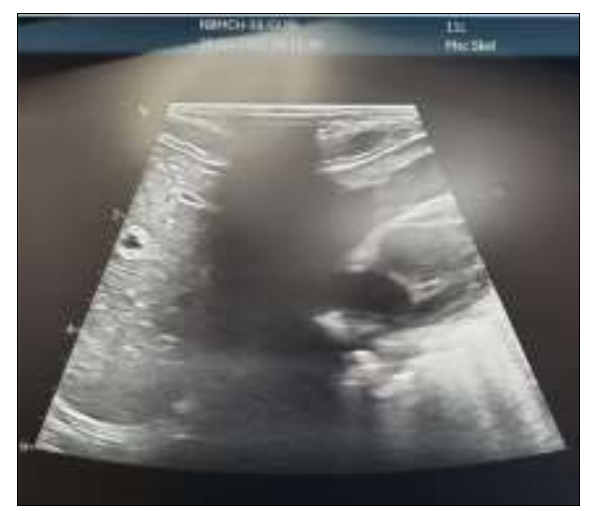

Fig 1: USG showing liver elevated from its normal position to the level of the nipple region on the right side adjacent to the heart

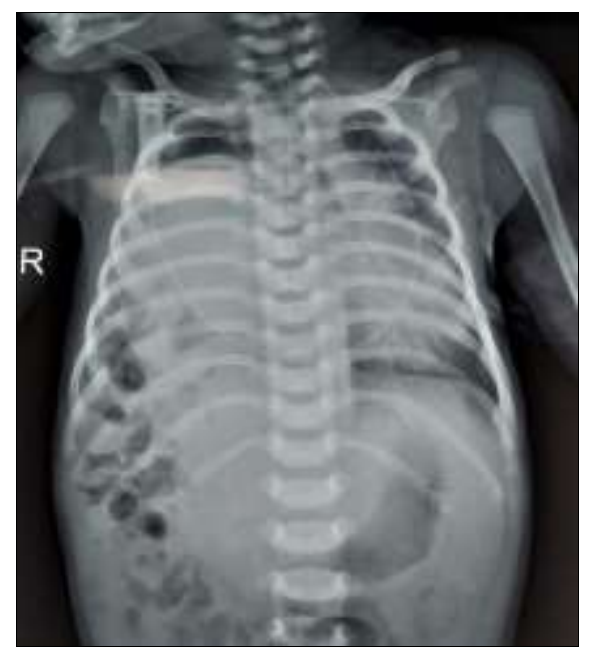

Fig 2: CXR AP supine showing gross volume loss of right hemithorax, liver herniating inside right hemithorax, and bowel inside right hemithorax

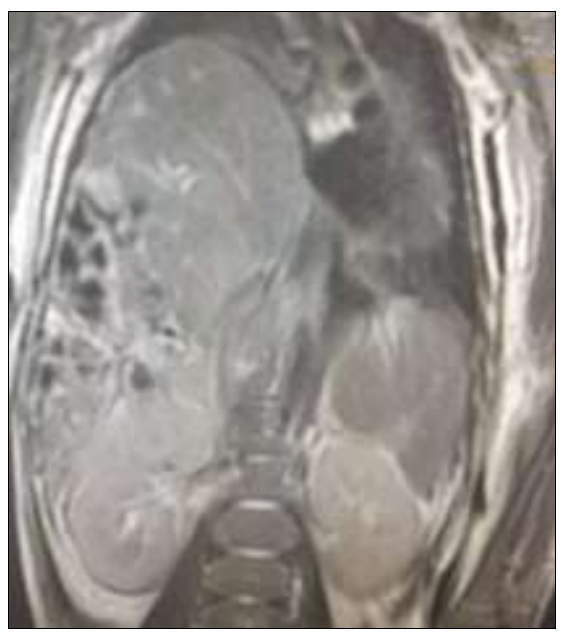

Fig 3: MRI shows gross elevation of the right hemidiaphragm along with the relatively higher position of the liver. No breach or

discontinuity in the contour of the diaphragm could be demonstrated

\section{Discussion}

The eventration of the diaphragm was first described in 1774. The first successful repair in an adult was done in 1923 by Jean Louis Petit. The first successful repair in an infant was done by Bisgard in 1946. It is a rare anomaly with an incidence of about5 in 10,000 live births. There is an increased prediction for the male sex and it is more common on the left ${ }^{[1]}$. Partial eventration is common in the anteromedial region of the right hemidiaphragm and complete presentation is commonly found in the left hemidiaphragm ${ }^{[2]}$. Eventration of the diaphragm may be congenital or acquired. Congenital eventration occurs when there is inadequate development of the muscle or absence of the phrenic nerves. The most common cause of acquired eventration is an injury to the phrenic nerve, resulting from either a birth trauma or thoracic surgery for congenital heart disease ${ }^{[3]}$. Eventration of the diaphragm is generally associated with pulmonary hypoplasia of varying degrees. It is due to the compression effect of the abdominal viscera on the developing lung. Respiratory distress is the most common clinical manifestation. However, findings vary depending on the extent of the anomaly. Patients with minor anomalies are generally asymptomatic, whereas extensive anomalies in neonates can cause respiratory distress ${ }^{[4]}$. Severe cases may need endotracheal intubation and mechanical ventilation support ${ }^{[5]}$. The exact etiology of congenital diaphragmatic eventration is unknown to date, although it postulated that it may be associated with fetal rubella or cytomegalovirus infection ${ }^{[5]}$. Sometimes, it is difficult or impossible to distinguish the entity from diaphragmatic paralysis and congenital diaphragmatic hernia. In eventration, the diaphragm retains its continuity and attachments to the boney skeleton. In a diaphragmatic hernia, there is a disruption in the continuity between the diaphragm and the costal margin ${ }^{[3]}$. The diaphragm is uniform in eventration but in a diaphragmatic hernia, there is generally a defect through which herniation of intraabdominal contents occurs. There is no pulmonary hypoplasia in eventration as compared to diaphragmatic hernia; therefore it is often undetected at birth and picked up as a coincidental finding ${ }^{[3,5]}$. Eventration generally remains asymptomatic early in life. Many patients present later in life with respiratory or very rarely gastrointestinal complaints ${ }^{[6]}$. Due to similarity in imaging findings, eventration is easily missed on plain radiographs for a congenital diaphragmatic hernia. It is important to distinguish the two conditions properly as they have different management protocols and different prognoses. USG in expert hands may be able to distinguish eventration from a diaphragmatic hernia. However, the modality of choice is MRI where a continuous hemidiaphragm on the affected side rules out a congenital diaphragmatic hernia. The treatment of choice is surgical plication. Thoracoscopic surgery is preferred over open surgery ${ }^{[7]}$.

\section{Conclusion}

Right-sided congenital diaphragmatic hernia and congenital eventration of the diaphragm are both rare disorders. Both these conditions can present similar features of respiratory compromise in a child. It is very easy to confuse one of these conditions for another due to their similar appearances on radiographs. MRI is the imaging modality of choice when confusion arises. 


\section{Declarations}

\section{Consent for publication}

The author clarifies that written informed consent was obtained from the parents and the anonymity of the patient was ensured.

\section{Competing interests}

The authors declare that they have no competing interests.

\section{Funding}

No funding resources.

\section{References}

1. Ozturk O, Yildız Y, Igde M, Oksuz BG. Coexistence of diaphragm eventration and thoracic ectopic kidney, Hong Kong Journal of Paediatrics 2015;20(1):37-40.

2. Bharadwaj PVV, Reddy VVR, Behra G, Praveen JV. Right-sided diaphragmatic eventration: a rare entity, IOSR Journal of Dental and Medical Sciences 2014;13(11):54-55.

3. Garcia-Prats JA. Eventration of the Diaphragm in Infants 2011.

4. Deslauriers J. Eventration of the diaphragm. Chest Surg Clin N Am 1998;8:315.

5. Barakat NA, Maaty SH, Al-Koly A. Outcome of Congenital Diaphragmatic Defects: 3 Years Experience. International Journal of Academic Research 2010;2:183-187.

6. Tiryaki T, Livanelioglu Z, Atayurt H. Eventration of the Diaphragm. Asian Journal of Surgery 2006;29:8-10. http://dx.doi.org/10.1016/S1015-9584(09)60285-2

7. Becmeur F, Talon I, Schaarschmidt K, Philippe P, Moog R, Kauffmann I, et al. Thoracoscopic Diaphragmatic Eventration Repair in Children: About 10 Cases. Journal of Pediatric Surgery 2005;40:17121715. http://dx.doi.org/10.1016/j.jpedsurg.2005.07.008. 